\title{
A coupled cluster study of Abelian lattice gauge field theories
}

Document Version

Accepted author manuscript

Link to publication record in Manchester Research Explorer

\section{Citation for published version (APA):}

Bishop, RF., Kendall, AS., Wong, LY., \& Xian, Y. (1993). A coupled cluster study of Abelian lattice gauge field theories. In L. Blum, \& F. B. Malik (Eds.), Condensed Matter Theories, Vol. 8 (pp. 269-290). Plenum Publishing Corporation. http://personalpages.manchester.ac.uk/staff/raymond.bishop/RFB_papers/[101] CMT_8(1993)269

\section{Published in:}

Condensed Matter Theories, Vol. 8

\section{Citing this paper}

Please note that where the full-text provided on Manchester Research Explorer is the Author Accepted Manuscript or Proof version this may differ from the final Published version. If citing, it is advised that you check and use the publisher's definitive version.

\section{General rights}

Copyright and moral rights for the publications made accessible in the Research Explorer are retained by the authors and/or other copyright owners and it is a condition of accessing publications that users recognise and abide by the legal requirements associated with these rights.

\section{Takedown policy}

If you believe that this document breaches copyright please refer to the University of Manchester's Takedown Procedures [http://man.ac.uk/04Y6Bo] or contact uml.scholarlycommunications@manchester.ac.uk providing relevant details, so we can investigate your claim.

\section{OPEN ACCESS}




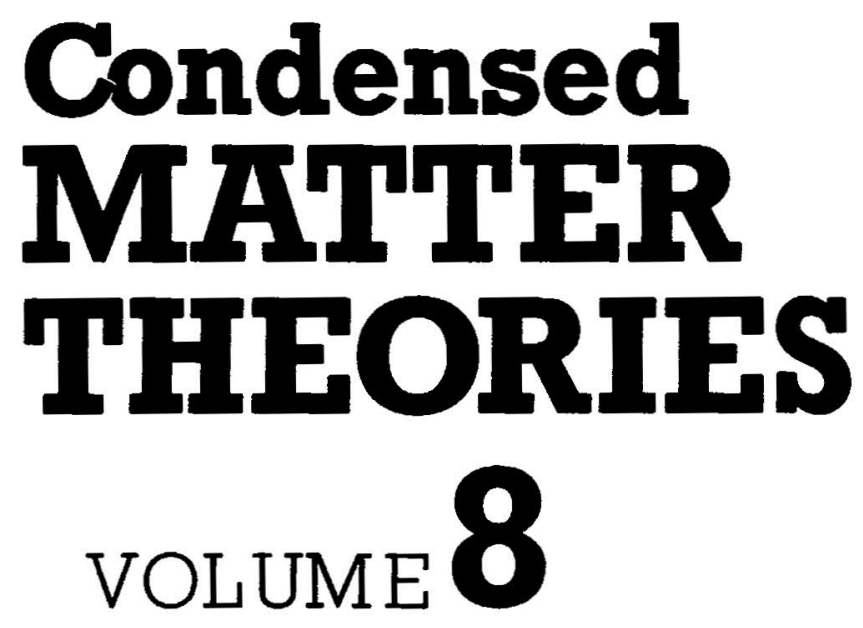

Edited by

Lesser Blum

University of Puerto Rico

Rio Piedras, Puerto Rico

and

F. Bary Malik

Southern Illinois University

Carbondale, Illinois

Plenum Press • New York and London 
Proceedings of the Sixteenth International Workshop on Condensed Matter Theories, held June 1-5, 1992, in San Juan, Puerto Rico

Library of Congress Catalog Card Number 87-656591 (ISSN 0893-861X)

ISBN 0-306-44405-4

(C) 1993 Plenum Press, New York

A Division of Plenum Publishing Corporation

233 Spring Street, New York, N.Y. 10013

All rights reserved

No part of this book may be reproduced, stored in a retrieval system, or transmitted in any form or by any means, electronic, mechanical, photocopying, microfilming, recording, or otherwise, without written permission from the Publisher

Printed in the United States of America 


\title{
A COUPLED CLUSTER STUDY OF ABELIAN LATTICE GAUGE FIELD THEORIES
}

\author{
R.F. Bishop, A.S. Kendall, L.Y. Wong and Yang Xian \\ Department of Mathematics, UMIST \\ (University of Manchester Institute of Science and Technology) \\ P.O. Box 88, Manchester M60 1QD, England
}

\section{INTRODUCTION}

It is widely accepted that the quantum chromodynamics (QCD) of interacting quarks and gluons is the best theory of the strong interaction, and hence of (sub-)hadronic structure, that is presently available. In particular, a full explanation of both the hadronic spectrum and quark confinement requires a careful treatment of this non-Abelian gauge field theory in the nonperturbative sector. A common approach to QCD is via the lattice formulation, 1,2 which involves an ultraviolet cutoff that both renders the theory well-defined and strictly preserves the important local gauge invariance. ${ }^{3}$

In view of the complicated structure of the non-Abelian QCD, it has seemed sensible to many people to study first various algebraically simpler Abelian gauge theories as a testing-ground for the theoretical tools to be used. In particular, both the continuous compact gauge group U(1) describing conventional quantum electrodynamics (QED),2,4-6 and the discrete gauge groups $(\mathrm{Z}(\mathrm{N})$; $N=$ $2,3, \cdots\}^{7,8}$ have been suggested and widely studied on the lattice in this context. The latter groups, which are simply the sets of complex Nth roots of unity with ordinary multiplication as the group operation, are interesting for a number of reasons. In the first place, as $N \rightarrow \infty$ the group $Z(N)$ passes smoothly over to U(1). Secondly, for $N=2$, the $Z(2)$ lattice gauge theory reduces to a gauge-invariant generalization of the Ising model, first considered by Wegner ${ }^{9}$ in the context of an investigation of condensed matter systems which exhibit phase transitions without possessing a local order parameter. Lastly, the $\mathrm{Z}(\mathrm{N})$ gauge theories are also closely connected to the spin-glass models of Edwards and Anderson. ${ }^{10}$

The close relationship of the $Z(2)$ gauge model to the Ising spin model noted above is an example of a rather remarkable, more general set of relationships or analogies first noted by Migdal. ${ }^{11}$ Thus, most (3+1)dimensional gauge field theories are similar to their $(1+1)$-dimensional spinchain system counterparts in a number of well-defined senses, as described in some detail by Fradkin and Susskind, ${ }^{5}$ for example. Perhaps the principal difference between such spin models as the Ising model and such gauge models as $\mathrm{Z}(2)$ is the fact that in the latter a symmetry is incorporated at the local level which is present in the former only globally. Thus, the Ising model is symmetric with respect to a reversal of the direction of the spins on all 
lattice sites, whereas in the $Z(2)$ model this symmetry is incorporated locally with respect to a definite site on the lattice. Thus, as explained in Sec. 2, the $Z(2)$ model Hamiltonian is invariant with respect to reversing the sign of only the spins connected to that site. All gauge symmetries, $1,2,7,9$ are local symmetries in this sense, namely of involving an invariance with respect to an operation affecting only degrees of freedom localized near some point.

As we have already indicated, lattice techniques have become the principal tool for treating the interesting nonperturbative physical content of gauge theories. Much of the effort to date has come from large-scale Monte Carlo studies of various kinds, 1,12-16 and other finite lattice calculations. ${ }^{17-20}$ Nevertheless, it is clearly also useful to apply such analytic tools as are available, in order to gain additional physical insight into the various gauge models. Amongst others, renormalization-group ideas, variational methods, weakand strong-coupling expansions, and Padé-approximant analysis have all been employed. However, with one principal exception, ${ }^{21}$ techniques from microscopic quantum many-body theory have largely been notable by their absence until now.

It is our contention that the application of ab initio many-body techniques to gauge field problems is especially timely for two distinct reasons. In the first place, the last decade has seen enormous progress in quantum many-body theory at both the formal level and in the range of successful applications made. Two methods in particular have proven themselves to be extremely versatile, able to achieve very high accuracy in practice, and capable of systematic improvement. These are the method of correlated basis functions ${ }^{22}$ (CBF) and the coupled cluster method 23,24 (CCM). They are widely recognized as providing the two most powerful, microscopic formulations currently available for dealing with $a b$ initio quantum many-body systems. Secondly, one of the founders of lattice gauge theory has recently voiced his strong pessimism and reservations about the developments still needed within lattice QCD before any really meaningful interaction with experiment can take place. ${ }^{25}$ In particular, he quantifies the existing gap between theory and experiment by suggesting that an increase in computing power of at least a factor of $10^{8}$ and equally powerful algorithmic or methodological advances are both needed.

It is also pertinent to note that as part of the above discussion Wilson recommends the lattice gauge community to look, for example, to the field of quantum chemistry for additional inspiration and new ideas, especially since both fields share a common concern with many-fermion systems interacting via long-range (unscreened) forces. What is valid for quantum chemistry in particular is clearly valid for many-body theory in general. Within this context we also note that one of the methods widely applied in modern $a b$ initio quantum chemistry is the CCM. For example, the very high accuracy required nowadays for the calculation of parity violation in atoms, as well as for the calculation of molecular energy differences of chemical significance calls for extreme accuracy in the solution of the electron correlation problem. ${ }^{26}$ The CCM is ideally suited for such applications. Indeed, it is now widely recognized as the method of first choice in terms of power and accuracy for calculations on, for example: ionization potentials, electron affinities, Auger spectroscopy, excitation energies, and energy gradients (for use, for example, in searching potential energy surfaces to predict vibrational spectra, or to locate transition states in decomposition reactions). A large number of atoms and molecules (including, e.g., $\mathrm{LiH}, \mathrm{H}_{2} \mathrm{O}, \mathrm{GaAs}$, benzene, etc.) has been studied, and state-of-the-art calculations are now being done on molecules with up to 80 active electrons. ${ }^{26}$

Finally, we note that the CCM has also recently been applied with considerable success to various spin-lattice problems. These include the solid 
phases of ${ }^{3} \mathrm{He},{ }^{27}$ and various models exhibiting antiferromagnetism. ${ }^{28}$ The latter includes the Heisenberg model on a two-dimensional square lattice, which is believed to be of relevance to the (undoped) ceramic cuprate materials exhibiting high-temperature superconductivity. In the light of all of the above comments, it seems especially worthwhile to attempt to apply similar CCM techniques to lattice gauge field theories. The purpose of the present paper is to outline our preliminary steps in this respect for the Abelian models $\mathrm{Z}(2)$ and $\mathrm{U}(1)$ in (2+1)-dimensions.

After outlining the two models themselves in Sec. 2, the formal aspects of the application of the CCM to them is described in Sec. 3. In order to implement the otherwise formally exact CCM in practice, approximation schemes must be introduced. The lowest-order member of a particular hierarchy of such truncation schemes which retains selected correlations of arbitrarily long range is described in Sec. 4 within the context of the U(1) model. A different, more localized, scheme is discussed in Sec. 5, where it is applied to the $Z(2)$ model. In both cases comparison is made with the results of perturbation theory and other methods. Our own results are summarized and discussed in Sec. 6 .

\section{THE U(1) AND Z(2) MODELS}

\subsection{The U(1) Lattice Hamiltonian}

The Hamiltonian for the free Maxwell field of electrodynamics in a $(d+1)-$ dimensional continuum of $\mathrm{d}$ spatial dimensions and one time dimension is given in the axial or temporal gauge (where $A_{0}=0$ ) by the usual expression,

$$
H=\frac{1}{2} \int d^{d} x\left[\vec{E}^{2}(x)+\vec{B}^{2}(x)\right]
$$

in terms of the electric field $\vec{E}=\vec{E}(x)$ and magnetic field $\vec{B}=\vec{B}(x)$. The magnetic field in turn is given in terms of the vector potential $\vec{A}=\vec{A}(x)$ by the usual relation,

$$
\overrightarrow{\mathrm{B}}=\vec{\nabla} \times \overrightarrow{\mathrm{A}}
$$

If the spatial continuum is now replaced by a d-dimensional hypercubic lattice, the discrete generalization of the vector potential field $\vec{A}(x)$ is the phase angle $A_{\ell} \in[-\pi, \pi]$ defined on the links $\{\ell\}$ joining nearest-neighbour lattice sites. In the usual Wilson ${ }^{1}$ formulation each link now has associated with it an element $\mathrm{U}_{\ell} \equiv \exp \left(\mathrm{iA}_{\ell}\right)$ of the underlying group $\mathrm{U}(1)$. If we set the lattice spacing to be unity we have $A_{\ell}=g \vec{\ell} \cdot \vec{A}$ where $\vec{\ell}$ is the directed vector joining the two sites of the link, and $g$ is the $U(1)$ coupling constant. The electric field operators are also defined on the links, $\hat{\ell} \cdot \vec{E}(x)=E_{\ell}(x) \rightarrow E_{\ell}$, and the quantization of the Hamiltonian on the lattice is now associated with the commutation relation,

$$
\left[\mathrm{A}_{\ell}, E_{\ell^{\prime}}\right]=\mathrm{i} \delta_{\ell \ell^{\prime}}
$$

Thus, the electric field operators $\left\{E_{\ell}\right\}$ are conjugate to the link phase angles, and can be represented as, 


$$
E_{\ell}=-i \frac{\partial}{\partial A_{\ell}}
$$

Just as in the continuum theory $\vec{A}(x)$ is not a gauge-invariant quantity, so nor are the equivalent link phases $A_{\ell}$ in the lattice case. However, we may introduce a gauge-invariant phase $B_{p}$ associated with each plaquette $p$ defined to be simply an elementary square formed of four links on the hypercubic lattice, by making a lattice generalization of Eq. (2). We thus define the group-element-valued plaquette variable $U_{p}$ as an oriented product of the link group elements bordering the plaquette as shown in Fig. 1,

$$
U_{p} \equiv \exp \left(i_{p}\right) \equiv U_{1} U_{2} U_{3}^{\dagger} U_{4}^{\dagger}=\exp \left[i\left(A_{1}+A_{2}-A_{3}-A_{4}\right)\right] .
$$

The lattice generalization of the magnetic energy density, $\frac{1}{2} \vec{B}^{2}(x)=\frac{1}{2}|\vec{\nabla} \times \vec{A}(x)|^{2}$, in the continuum expression of Eq. (1) is now simply,

$$
\begin{aligned}
\underset{2}{\frac{1}{B^{2}}(x) \rightarrow} & \frac{1}{\mathbf{g}^{2}}\left[1-\frac{1}{2}\left(U_{p}+U_{p}^{\dagger}\right)\right] \\
& =\lambda\left(1-\cos B_{p}\right),
\end{aligned}
$$

where $\lambda \equiv \mathrm{g}^{-2}$ is the non-negative dimensionless coupling constant. The strongcoupling limit is defined by $\lambda \rightarrow 0(\mathrm{~g} \rightarrow \infty)$, and the weak-coupling limit by $\lambda \rightarrow \infty$ $(g \rightarrow 0)$. We thus have the $\mathrm{U}(1)$ lattice Hamiltonian analogue of Eq. (1),

$$
\mathbf{H}=-\frac{1}{2} \sum_{\ell} \frac{\partial^{2}}{\partial A_{\ell}^{2}}+\lambda \sum_{\mathrm{P}}\left(1-\cos \mathrm{B}_{\mathrm{p}}\right)
$$

where the periodicity in the magnetic term plays a crucial role in maintaining the true phase structure of the theory. ${ }^{4}$

Since the physical states are gauge-invariant, ${ }^{3}$ we may equivalently write the Hamiltonian of Eq. (7) wholly in terms of the plaquette phases $\left\{B_{p}\right\}$, rather than partially using the gauge-dependent link phases $\left\{A_{\ell}\right\}$, provided we restrict $H$ to operate only on physical states. By making use of Eq. (5), we can thus easily express $H$ in terms of the plaquette variables alone for any dimensionality $d$. We quote the result only for the $d=2$ (square lattice case),



Fig. 1. Schematic definition of the plaquette variable $p$ by the ordered product of the 4 links defining it. 


$$
H=\sum_{p}\left[-2 \frac{\partial^{2}}{\partial B_{p}^{2}}+\lambda\left(1-\cos B_{p}\right)\right]+\frac{1}{2} \sum_{p} \sum_{\rho} \frac{\partial^{2}}{\partial B_{p} \partial B_{p}+\rho}
$$

where $\rho$ is a lattice vector connecting neighbouring plaquettes on the twodimensional (2D) lattice. The Hamiltonian of $\mathrm{Eq}$. (8) is invariant under the transformation $B_{p} \rightarrow B_{p}+2 n \pi$, with $n$ integral. Hence the space of $B_{p}$ is compact $\left(-\pi \leq \mathrm{B}_{\mathrm{p}} \leq \pi\right.$, for all $\left.\mathrm{p}\right)$, and the Hamiltonian is called that of compact (or periodic) QED.

We note that in the $(2+1)$-dimensional case studied here, compact QED is known to be asymptotically free and not to undergo a deconfining phase transition. Perturbative results are available in the strong-coupling regime, ${ }^{29}$ whilst the weak-coupling limit has mainly been studied via the villain ${ }^{30}$ approximation as applied to the Euclidean lattice.31,32 Monte Carlo techniques have also been applied to the 2D lattice. ${ }^{14,33}$ Other treatments have included various variational approaches ${ }^{34,35}$ and their systematic improvement via the Lanczos algorithm. ${ }^{36,37}$ We shall make a comparison of our own results with those from some of these alternative methods in Sec. 4.

\subsection{The $Z(2)$ Lattice Hamiltonian}

As in the $U(1)$ case above, the $Z(N)$ models are defined by a one-parameter $(\lambda)$ family of Hamiltonians specified in terms of operators $\left\{\mathrm{P}_{\ell}, Q_{\ell}\right\}$ associated with the links $\{\ell\}$ of a hypercubic lattice in $d$ spatial dimensions. These are defined to obey the $\mathrm{Z}(\mathrm{N})$ algebra,

$$
\begin{gathered}
\mathrm{P}_{\ell}^{N}=\mathrm{Q}_{\ell}^{N}=1, \quad \mathrm{P}_{\ell}^{+} \mathrm{P}_{\ell}=\mathrm{Q}_{\ell}^{\dagger} \mathrm{Q}_{\ell}=1, \\
\mathrm{P}_{\ell} \mathrm{Q}_{\ell}=\exp (-2 \pi \mathrm{i} / \mathrm{N}) \mathrm{Q}_{\ell} \mathrm{P}_{\ell} .
\end{gathered}
$$

Operators on different links commute. It follows from Eq. (9) that $P_{\ell}$ and $Q_{\ell}$ are ladder operators with respect to one another. For example, in a representation in which $\mathrm{P}_{\boldsymbol{\ell}}$ is diagonalized,

$$
P_{\ell}|L\rangle=\exp (-2 \pi i L / N)|L\rangle
$$

the operator $Q_{\ell}$ has the following mode of action,

$$
\mathrm{Q}_{\ell}|\mathrm{L}\rangle=|\mathrm{L}+1\rangle ; \quad|\mathrm{L}+\mathrm{N}\rangle=|\mathrm{L}\rangle \text {. }
$$

Equivalently, just as in the U(1) case each link $\ell$ has associated with it an element $U_{\ell}$ of the group $Z(N)$ defined by,

$$
\mathrm{Z}(\mathrm{N})=\left\{\mathrm{e}^{\mathrm{in} \delta} ; \quad \delta \equiv \frac{2 \pi}{\mathrm{N}} ; \mathrm{n}=1,2, \cdots, \mathrm{N}\right\}
$$

Clearly, for the case $N=2$ considered here, the algebra of Eq. (9) can be realized by choosing $P_{\ell}$ and $Q_{\ell}$ to be any two of the usual Pauli matrices, $\sigma_{1}(\ell)$, $i=1,2,3$, defined on the links $\{\ell\}$. We make here the (standard) choice of $\sigma_{1}(\ell)$ and $\sigma_{3}(\ell)$.

For reasons explained more fully elsewhere, $5,8,38$ the quantum $Z(N)$ Hamiltonian is taken to be, 


$$
\mathrm{H}=\frac{1}{2} \sum_{\ell}\left(2-\mathrm{P}_{\ell}^{+}-\mathrm{P}_{\ell}\right)-\frac{1}{2} \lambda \sum_{\mathrm{P}}\left(\mathrm{Q}_{1} \mathrm{Q}_{2} \mathrm{Q}_{3}^{+} \mathrm{Q}_{4}^{+}+\text {H.c. }-2\right),
$$

where the second term involves a sum over the elementary plaquettes of the oriented product of the link elements $\left\{Q_{\ell}\right\}$ bordering the plaquette, as shown in Fig. 1. The $N \rightarrow \infty$ limit is readily related to the $U(1)$ model above, after a trivial re-scaling of the first term, by making the following representations of the operators $\mathrm{P}_{\ell}$ and $\mathrm{Q}_{\ell}$,

$$
\left.\mathrm{P}_{\ell}=\exp \left(-\frac{2 \pi \mathrm{i}}{\mathrm{N}} \mathrm{E}_{\ell}\right), \mathrm{Q}_{\ell}=\exp (\mathrm{iA})_{\ell}\right)
$$

where $E_{\ell}$ and $A_{\ell}$ are dimensionless Hermitian operators associated with the link $\ell$. They are the analogues of the electric field and vector potential operators, and they obey the commutation relation of Eq. (3). Further details are given in Ref. [8].

Henceforth, we restrict ourselves to the $\mathrm{Z}(2)$ model, which we can thus represent (after the omission of some trivial constant terms) in terms of spin variables in the form,

$$
H=-\sum_{\ell} \sigma_{1}(\ell)-\lambda \sum_{\mathrm{p}} \sigma_{3}(1) \sigma_{3}(2) \sigma_{3}(3) \sigma_{3}(4),
$$

in terms of the plaquette variables defined in Fig. 1. The Hamiltonian of Eq. (15) has a local gauge invariance as we now demonstrate. A local gauge transformation at a specific lattice site $\mathrm{n}$ is brought about by the application of the operator $\mathrm{G}(\mathrm{n})$ defined as,

$$
G(n)=\prod_{1=1}^{2 d} \sigma_{1}\left(\ell_{n ; 1}\right)
$$

where the product is over all $2 \mathrm{~d}$ links $\left\{\ell_{\mathrm{n} ; \mathrm{i}} ; \mathrm{i}=1,2, \cdots, 2 \mathrm{~d}\right\}$ attached to the site $n$. The operator $G(n)$ is unitary, and its mode of action on the spin operators is as follows,

$$
\begin{aligned}
& G^{-1}(n) \sigma_{1}(\ell) G(n)=\sigma_{1}(\ell) ; \text { all } \ell, \\
& G^{-1}(n) \sigma_{3}(\ell) G(n)=\left\{\begin{array}{l}
-\sigma_{3}(\ell) ; \ell \text { attached to } n, \\
\sigma_{3}(\ell) ; \ell \text { not attached to } n .
\end{array}\right.
\end{aligned}
$$

Thus the application of $\mathrm{G}(\mathrm{n})$ changes the sign of the $\sigma_{3}$ operators attached to site $\mathrm{n}$, and leaves all other $\sigma_{1}$ and $\sigma_{3}$ operators unchanged. Clearly, the Hamiltonian of Eq. (15) commutes with all $G(n)$, as it has been constructed from products of $\sigma_{3}$ operators taken around closed loops (i.e., elementary plaquettes), and hence $H$ is left invariant under all $G(n)$.

The ground state of the Hamiltonian of Eq. (15) may also be proven ${ }^{3}$ to be invariant under these gauge transformations. Since we are interested only in gauge-invariant operators acting on the ground state, all of the states $\{|\Psi\rangle\}$ of physical interest are hence gauge-invariant,

$$
G(n)|\Psi\rangle=|\Psi\rangle \text {; all } n \text {. }
$$


As a consequence, Eq. (18) immediately implies that the expectation value in any gauge-invariant state of any operator $A$ which is not gauge-invariant [i.e., $G^{-1}(n) A G(n) \neq A$ for all $n$ l must vanish identically. In particular, there can be no nonzero magnetization in any state satisfying gauge invariance, and no phase transition can lead to a magnetized phase. Nevertheless, as Wegner ${ }^{9}$ first pointed out, the $\mathrm{Z}(2)$ lattice models for various values of the dimensionality $d$ do exhibit phase transitions, as we discuss below.

The above is a particular example of Elitzur's theorem ${ }^{3}$ that a local symmetry cannot break down spontaneously. In this respect such gauge field theories and their local invariance properties are very different from the global invariance properties of, for example, the related Ising model, whose Hamiltonian is invariant only under a change of the sign of all of the spin operators simultaneously. In that case, the ground state of the Ising model is doubly degenerate and the symmetry transformation takes one vacuum to the other. The difference in the stability properties of the spontaneously broken symmetry states in the two cases has especially been stressed by Fradkin and Susskind. ${ }^{5}$

Finally, we note that certain well-defined duality relations are known for the $\mathrm{Z}(2)$ model.5,7-9 For the two-dimensional lattice $(\mathrm{d}=2)$ the theory is dual to the Ising model in two spatial dimensions in a transverse magnetic field, and is thus a two-phase system which undergoes a second-order phase transition at some critical coupling, $\lambda=\lambda_{c}$. Estimates of $\lambda_{c}$ have been obtained by various means, including perturbation-theory expansions, ${ }^{39} 1 / \mathrm{N}$-expansions, ${ }^{40}$ finite lattice studies, ${ }^{17,18}$ renormalization-group techniques, ${ }^{41}$ and the application ${ }^{42}$ of a perturbative-variational method due to Bessis and Villani.43 The first two of these are among the most accurate. They give the respective values $\lambda_{c} \approx 3.08$ and $\lambda_{c} \approx 3.055$. By contrast, for $d=3$ the $Z(2)$ model is self-dual with a symmetry point at $\lambda=1$. Free energy expansions ${ }^{7}$ have indicated that the $d=3$ system is a two-phase system with a first-order phase transition at $\lambda=1$. Monte Carlo simulations ${ }^{13}$ and all other subsequent work have provided confirmation of this.

\section{A COUPLED CLUSTER TREATMENT OF THE U(1) AND Z(2) MODELS}

Since the CCM has been well reviewed many times in the past, we refer the reader to the literature for both a general description of the method (and see, for example, Ref. [24] and the references cited therein) and its previous applications to spin lattice problems in particular. ${ }^{28}$ We concentrate in this section on the specific applications to the two lattice gauge models described in Sec. 2.

\subsection{The $U(1)$ Model}

In order to describe the correlations in a many-body system the CCM starts from a suitable reference state. This is usually (but not always) the properly symmetrized, non-interacting many-body state composed of some single-particle wave functions chosen to be the eigenstates of an appropriate one-body Hamiltonian. The correlations present in the physical system are then incorporated via many-body excitations from this reference state. In this respect the CCM is similar to perturbation theory. Nevertheless, the CCM is highly nonperturbative, since the correlation operator actually takes the form of an exponentiated function, 23,24 as is by now rather well known, and as we shall see in detail below.

The Schrödinger equation for the $U(1)$ Hamiltonian of Eq. (8) reduces in the 
case of a single plaquette to the Mathieu equation,

$$
-2 \frac{\mathrm{d}^{2}}{\mathrm{~dB}^{2}} \psi_{\mathrm{n}}(\mathrm{B})+\lambda(1-\cos \mathrm{B}) \psi_{\mathrm{n}}(\mathrm{B})=\varepsilon_{\mathrm{n}} \psi_{\mathrm{n}}(\mathrm{B}) ;-\pi \leq \mathrm{B} \leq \pi
$$

In view of the fact that the Mathieu equation is well-known to be highly nonperturbative, it is pertinent to consider the simpler problem of its strongcoupling $(\lambda \rightarrow 0)$ limit first. This (strong-coupling) unperturbed Hamiltonian,

$$
\mathrm{H}_{0}=-2 \mathrm{~d}^{2} / \mathrm{dB}^{2} ;-\pi \leq \mathrm{B} \leq \pi,
$$

has two sets of eigenstates, namely $\{\cos \mathrm{mB} ; \mathrm{m}=0,1,2, \cdots\}$ with even parity, and ( $\sin \mathrm{mB} ; \mathrm{m}=1,2, \cdots$ \} with odd parity. The ground state of $\mathrm{H}_{0}$ is clearly just a constant. We now use these simple strong-coupling single-plaquette wave functions to construct both the ket and bra ground states in terms of a CCM analysis.

The non-interacting reference state $|\Phi\rangle$ is hence simply a constant, in which all plaquettes are described by the ground state (g.s.) of $\mathrm{H}_{0}$. The exact many-body ket g.s. of the U(1) Hamiltonian of Eq. (8) is then taken in the CCM form,

$$
\left|\Psi_{o}\left(\left\{B_{p}\right\}\right)\right\rangle=e^{S\left(\left\{B_{p}\right\}\right)}|\Phi\rangle, S\left(\left\{B_{p}\right\}\right)=\sum_{k=1}^{N_{p}} S_{k}\left(\left\{B_{p}\right\}\right),
$$

where $\mathrm{N}_{\mathrm{p}}$ is the total number of plaquettes in the lattice and where the $\mathrm{k}$-body correlation operators $S_{k}=S_{k}\left(\left\{B_{p}\right\}\right)$ are decomposed as,

$$
\begin{gathered}
S_{1}=\sum_{n=1}^{\infty} \sum_{p=1}^{N} s_{p}(n) \cos \left(n B_{p}\right), \\
S_{2}=\frac{1}{2 !} \sum_{n_{1}, n_{2}=1}^{\infty} \sum_{p_{1}, p_{2}=1}^{N}\left[s_{p_{1} p_{2}}^{(1)}\left(n_{1}, n_{2}\right) \cos \left(n_{1} B_{p_{1}}\right) \cos \left(n_{2} B_{p_{2}}\right)\right. \\
\left.\quad+s_{p_{1} p_{2}}^{(2)}\left(n_{1}, n_{2}\right) \sin \left(n_{1} B_{p_{1}}\right) \sin \left(n_{2} B_{p_{2}}\right)\right],
\end{gathered}
$$

where the prime on the summation in Eq. (22b) excludes the term $\mathrm{p}_{1}=\mathrm{p}_{2}$; and similarly for the higher-order partitions $S_{\mathbf{k}}$ with $k>2$. For reasons which have been described many times in the past ${ }^{24}$ the bra g.s., $\left\langle\tilde{\Psi}_{\circ}\right|$, in the CCM parametrization is not taken as the manifest Hermitian conjugate of $\left|\Psi_{0}\right\rangle$. Instead it is parametrized in the form,

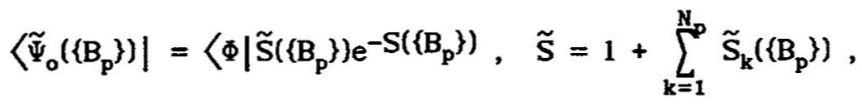

where $\mathbf{S}=\mathbf{S}\left(\left\{\mathrm{B}_{\mathrm{p}}\right\}\right)$ is as given above in Eqs. (21)-(22), and $\tilde{\mathbf{S}}=\tilde{\mathbf{S}}\left(\left\{\mathrm{B}_{\mathrm{p}}\right\}\right)$ is similarly defined as,

$$
\tilde{S}_{1}=\sum_{n=1}^{\infty} \sum_{p=1}^{N_{p}} \tilde{S}_{p}(n) \cos \left(n B_{p}\right)
$$




$$
\begin{aligned}
& \tilde{S}_{2}=\frac{1}{2 !} \sum_{n_{1}, n_{2}=1}^{\infty} \sum_{p_{1}, p_{2}=1}^{N}\left[\widetilde{s}_{P_{1} p_{2}}^{(1)}\left(n_{1}, n_{2}\right) \cos \left(n_{1} B_{p_{1}}\right) \cos \left(n_{2} B_{p_{2}}\right)\right. \\
& \left.+\widetilde{s}_{\mathbf{p}_{1} \mathrm{P}_{2}}^{(2)}\left(\mathrm{n}_{1}, \mathrm{n}_{2}\right) \sin \left(\mathrm{n}_{1} \mathrm{~B}_{\mathrm{p}_{1}}\right) \sin \left(\mathrm{n}_{2} \mathrm{~B}_{\mathrm{p}_{2}}\right)\right],
\end{aligned}
$$

and similarly for the higher-order terms. We note that both $\left|\Psi_{0}\right\rangle$ and $\left\langle\tilde{\Psi}_{0}\right|$ are explicitly invariant under the transformation $\mathbf{B}_{\mathbf{p}} \rightarrow \mathbf{B}_{\mathbf{p}}+2 \pi$. As befits the ground state, they have also been constructed to have even parity under interchange of the sign of all of the coefficients $\left\{B_{p}\right\}$.

We define an inner product of wave functions $\left\langle\widetilde{g}\left(\left\{B_{p}\right\}\right)\right|$ and $\left|f\left(\left\{B_{p}\right\}\right)\right\rangle$ as,

$$
\langle\widetilde{g} \mid f\rangle=\int_{-\pi}^{\pi} \prod_{p=1}^{N_{p}}\left(\frac{d_{p}}{2 \pi}\right) \tilde{g} \cdot f
$$

and the expectation value of an operator $\theta=\theta\left(\left\{B_{p}\right\},\left\{d / d B_{p}\right\}\right)$ with respect to the conjugate states $|f\rangle$ and $\langle\tilde{f}|$ as,

$$
\bar{\theta} \equiv\langle\tilde{f}|\theta| f\rangle=\int_{-\pi}^{\pi} \prod_{p=1}^{N_{p}}\left(\frac{d_{p}}{2 \pi}\right) \tilde{f} \cdot \theta \cdot f .
$$

The normalization condition, $\langle\Phi \mid \Phi\rangle=1$, then gives that $\Phi$ is equal to unity. The CCM equations for the correlation coefficients of Eqs. (22) and (24) are now derived from the condition that the g.s. expectation value of the Hamiltonian of Eq. (8),

$$
\overline{\mathrm{H}}=\left\langle\tilde{\Psi}_{\mathrm{o}}|\mathrm{H}| \Psi_{\mathrm{o}}\right\rangle=\left\langle\Phi\left|\tilde{S}^{-S_{H e}} \mathbf{S}\right| \Phi\right\rangle,
$$

should be stationary with respect to variations in both complete sets of braand ket-state coefficients. Thus, for example, the one-body coefficients $\left\{\widetilde{s}_{p}(n)\right\}$ and $\left\{s_{p}(n)\right\}$ are derived from the variational principle,

$$
\frac{\delta \bar{H}}{\delta \widetilde{S}_{p}(n)}=0=\frac{\delta \bar{H}}{\delta s_{p}(n)} .
$$

The equations for the two- and higher-body coefficients are similarly given by the corresponding stationarity conditions for $\overline{\mathrm{H}}$.

Within the context of the above CCM parametrization there are clearly two distinct kinds of correlations in play. In the first place one has the modecoupling terms between different modes $\left\{\cos \left(\mathrm{nB}_{\mathrm{p}}\right), \sin \left(\mathrm{nB}_{\mathrm{p}}\right)\right\}$ specified by the index $n$. Secondly, we have the more physical correlations between different plaquettes specified by the indices $\left\{p_{1}\right\}$. Clearly mode-coupling is included even at the one-plaquette level (i.e., as specified by $S_{1}$ and $\tilde{s}_{1}$ alone), whereas one needs to include at least $S_{2}$ and $\tilde{S}_{2}$ as well in order to describe plaquette correlations.

We also note that the CCM correlation operators of Eqs. (21)-(24) do not involve the usual creation and destruction operators as in the more 
conventional CCM. ${ }^{24,28}$ This distinct difference is related essentially to the fact that in the lattice gauge systems under consideration there are neither any real particles nor quasiparticles defined. Whereas in more conventional many-body problems one is usually concerned with particle conservation, the primary concern in lattice gauge models is the gauge invariance.

\subsection{The $Z(2)$ Model}

The strong-coupling $(\lambda \rightarrow 0)$ limit of the $Z(2)$-model Hamiltonian of Eq. (15) is

$$
\mathrm{H}_{0}=-\sum_{\ell} \sigma_{1}(\ell),
$$

where the sum is over all links. The model state $|\Phi\rangle$ of the CCM is now taken for this $\mathrm{Z}(2)$ gauge theory as the g.s. of Eq. (28), namely the state with all spins aligned with respect to the 1-axis, such that,

$$
\sigma_{1}(\ell)|\Phi\rangle=|\Phi\rangle ; \text { all } \ell
$$

Exactly as for the U(1) model, the ket and bra ground states $\left|\Psi_{0}\right\rangle$ and $\left\langle\tilde{\Psi}_{0}\right|$ of Eq. (15) are formed in terms of the correlation operators $\mathbf{S}$ and $\tilde{\mathrm{S}}$, as before. We recall that these states must be gauge-invariant with respect to the transformations induced by the operators $G(n)$ of Eq. (16). Since $|\Phi\rangle$ is also gauge-invariant, hence so must $\mathrm{S}$ and $\tilde{\mathrm{S}}$ be. As explained in Sec. 2.2 the easiest way to ensure this is to construct them from products of $\sigma_{3}$ operators taken around closed loops on the lattice. One way to achieve this is in terms of the elementary plaquette operators $\{U(\mathrm{p})\}$ defined as products around the links bordering the plaquettes as in Fig. 1,

$$
U(p)=\sigma_{3}(1) \sigma_{3}(2) \sigma_{3}(3) \sigma_{3}(4) \text {. }
$$

Hence, we may write for the ket g.s., by analogy with Eq. (21),

$$
\left|\Psi_{0}\right\rangle=e^{S}|\Phi\rangle, \quad S=\sum_{k=1}^{N_{p}} S_{k},
$$

where $\mathrm{N}_{\mathrm{p}}$ is the total number of plaquettes, and where the k-body partitions of the correlation operator are specified as,

$$
S_{k}=\frac{1}{k !} \sum_{p_{1}=1}^{N} \cdots \sum_{p_{k}=1}^{N_{p}} s_{p_{1} p_{2}} \cdots p_{k} U\left(p_{1}\right) \cdots U\left(p_{k}\right)
$$

where the primes on the summations indicate that only such terms are included where no two or more of the indices $\left(p_{1}, p_{2}, \cdots, p_{k}\right)$ are equal. The bra g.s. is similarly specified, by analogy with Eq. (23), in the form,

$$
\left\langle\tilde{\Psi}_{0}\right|=\langle\Phi| \tilde{S} e^{-S}, \tilde{S}=1+\sum_{k=1}^{N} \tilde{S}_{k},
$$

and where $\tilde{\mathrm{S}}_{\mathrm{k}}$ is parametrized exactly as in Eq. (32), except that $\left\{\mathrm{s}_{\mathrm{P}_{1}} \cdots_{\mathrm{P}_{\mathbf{k}}}\right\} \rightarrow$ $\left\{\tilde{s}_{p_{1}} \cdots p_{\mathbf{k}}\right\}$. 


\section{THE SUBI SCHEME FOR THE U(1) MODEL}

In principle, the exact ket and bra ground states of our U(1) lattice gauge model are given by Eqs. (21)-(24), where all correlation operators $\left\{S_{k}, \tilde{S}_{k} ; k=1,2, \cdots, N_{p}\right\}$ up to those between all $N_{p}$ plaquettes should be included. However, in practice one clearly needs to approximate. The most common CCM truncation scheme is the so-called SUBn scheme, ${ }^{24}$ in which only those correlations described by the cluster partitions $\left\{S_{k}, \tilde{S}_{k}\right\}$ with $k \leq n$ are included, and those with $\mathrm{k}>\mathrm{n}$ are set to zero. We focus preliminary attention here on the lowest-order SUBI approximation, for which we therefore make the replacements $\mathrm{S} \rightarrow \mathrm{S}_{1}, \tilde{\mathrm{S}} \rightarrow \widetilde{\mathrm{S}}_{1}$.

The retained coefficients $\left\{s_{p}(n), \widetilde{s}_{p}(n)\right\}$ are then found as described in Sec. 3.1, and as given by Eqs. (26) and (27). In particular, the SUB1 g.s. ket-coefficients are clearly obtained by solving the coupled set of equations,

$$
\left\langle\cos \left(\mathrm{mB}_{\mathrm{p}}\right)\left|\mathrm{e}^{-\mathrm{S}_{1_{\mathrm{He}}} \mathrm{S}_{1}}\right| \Phi\right\rangle=0 ; \mathrm{m}=1,2, \cdots,
$$

where the matrix element is defined as in Eq. (25b) and where, as described in Sec. 3.1, the model state $|\Phi\rangle$ is simply a constant, chosen to be unity so that $\langle\Phi \mid \Phi\rangle=1$ according to the inner product definition of Eq. (25a). Although the evaluation of the g.s. expectation value of an arbitrary operator in this SUB1 approximation would also require the bra g.s. coefficients $\left\{\widetilde{s}_{\mathbf{p}}(\mathbf{n})\right\}$, the g.s. energy, $E_{g}$, is clearly given, in view of Eqs. (23), (26) and (34), by the stationary value of $\overline{\mathrm{H}}$, and hence purely in terms of the ket g.s. coefficients as,

$$
E_{g}=\left\langle\Phi\left|e^{-S_{1}} \mathrm{He}^{S_{1}}\right| \Phi\right\rangle
$$

In terms of the parametrization of Eq. (22a), and using the explicit definition of the $(2+1)$-dimensional Hamiltonian in Eq. (8), it is not difficult to derive the explicit SUB1-approximation equations,

$$
\begin{gathered}
\sum_{p=1}^{N}\left[-\frac{1}{2} \lambda \delta_{m, 1}+m^{2} s_{p}(m)+\frac{1}{2} \sum_{n, n^{\prime}=1}^{\infty} n n^{\prime} s_{p}(n) s_{p}\left(n^{\prime}\right)\left(\delta_{m, n+n^{\prime}}-\delta_{m, l n-n^{\prime}}\right)\right] \\
=0 ; m=1,2, \cdots, \\
E_{g}=\lambda N_{p}-\sum_{p=1}^{N} \sum_{n=1}^{\infty} n^{2} s_{p}^{2}(n) .
\end{gathered}
$$

In the limit of an infinite lattice $\left(\mathrm{N}_{\mathrm{p}} \rightarrow \infty\right)$ considered here the coefficients $s_{p}(n)$ are independent of plaquette index $p$ by lattice translational invariance. Hence we may write,

$$
a_{m} \equiv m s_{p}(m) ; \quad m=1,2, \cdots \text {. }
$$


Furthermore, if we extend the definition of the coefficients $\left\{a_{m}\right\}$ to nonpositive integers by the relation,

$$
a_{-m}=-a_{m} ; m=0,1,2, \cdots,
$$

so that $a_{0} \equiv 0$, we may readily rewrite Eqs. (36) and (37) as,

$$
\left(\frac{E_{g}}{N_{p}}-\lambda\right) \delta_{m, 0}+\frac{1}{2} \lambda\left(\delta_{m, 1}+\delta_{m,-1}\right)-m a_{m}-\frac{1}{2} \sum_{n=-\infty}^{\infty} a_{n} a_{m-n}=0,
$$

which is valid for all integers $\mathrm{m}$. We may now define an odd function, $A=A(B)$, by the Fourier sum,

$$
A(B) \equiv \sum_{m=-\infty}^{\infty} a_{m} \sin (m B)
$$

whose inverse, taking into account the symmetry relation (39), is given by,

$$
a_{m}=\frac{1}{2 \pi} \int_{-\pi}^{\pi} d B A(B) \sin (m B)
$$

It is easy to show that Eq. (40) is equivalent to the first-order Riccati equation,

$$
\frac{d A}{d B}-\frac{1}{2} A^{2}=E_{g} / N_{p}-\lambda(1-\cos B)
$$

Finally, the standard substitution,

$$
\mathrm{A}=-\frac{2}{\psi} \frac{\mathrm{d} \psi}{\mathrm{dB}}
$$

reduces Eq. (42) to the Mathieu equation,

$$
\left[-2 d^{2} / d B^{2}+\lambda(1-\cos B)\right] \psi(B)=\left(E_{g} / N_{p}\right) \psi(B)
$$

which may be compared with the single-plaquette equation (19).

The fact that we regain the Mathieu equation in our SUB1 scheme is not too surprising since no multi-plaquette correlation effects have been included, and as we and others ${ }^{15,44}$ have shown, the single-plaquette problem is equivalent to the Mathieu equation. Nevertheless, the many-body nature of our system has not been entirely lost, since the eigenvalue in Eq. (44) is the intensive quantity, $E_{g} / N_{p}$. Furthermore, one can without too much difficulty go beyond this SUB1 level to include 2-plaquette (and higher-order) correlations in our CCM formalism.

For present purposes, however, we remain at the SUB1 level. In order to solve Eq. (40) it is natural to define the so-called SUB1(n) sub-hierarchy in which one retains at the $n^{\text {th }}$ level of approximation only those coefficients $a_{m}$ with which one retains at the $n^{\text {th }}$ level of approximation only those coefficients $a_{m}$ with $|m| \leq n$, and sets the remainder with $|m|>n$ to zero. Thus, in the SUB(1) scheme, only the single independent coefficient $a_{1}\left(=-a_{-1}\right)$ 
Table 1. The ground-state energy per plaquette, $E_{g} / N_{p}$, of the $U(1)$ gauge field model in (2+1)-dimensions, for various values of the coupling constant $\lambda$. Results in various SUBI(n) sub-approximations are shown.

\begin{tabular}{lcccccc}
\hline & \multicolumn{7}{c}{$\lambda$} \\
\cline { 2 - 7 } Method & 0.1 & 0.5 & 1.0 & 2.0 & 5.0 & 20.0 \\
\hline SUB1(1) & 0.097500 & 0.437500 & 0.750000 & 1.000000 & -1.25000 & -80.0000 \\
SUB1(2) & 0.097503 & 0.439123 & 0.772689 & 1.249117 & 2.209359 & 5.525510 \\
SUB1(3) & 0.097503 & 0.439117 & 0.772425 & 1.242552 & 2.061439 & 0.553501 \\
SUB1(4) & 0.097503 & 0.439117 & 0.772431 & 1.243018 & 2.099494 & 4.273716 \\
SUB1(6) & 0.097503 & 0.439117 & 0.772431 & 1.243022 & 2.099907 & 4.302545 \\
SUB1(8) & 0.097503 & 0.439117 & 0.772431 & 1.243021 & 2.099974 & 4.335206 \\
SUB1(20) & 0.097503 & 0.439117 & 0.772431 & 1.243021 & 2.099977 & 4.343306 \\
& & & & & & \\
\hline
\end{tabular}

is retained. The solution is trivially given as,

$$
a_{1}=-\frac{1}{2} \lambda, \quad E_{g} / N_{p}=\lambda-\frac{1}{4} \lambda^{2} ; \operatorname{SUB}(1) .
$$

Similarly in the SUB1(2) approximation one retains only the two independent coefficients $a_{1}$ and $a_{2}$. The solution to Eq. $(40)$ in this case is given by,

$$
\begin{aligned}
& a_{1}=\left(\sqrt{\lambda^{2}+64 / 27}+\lambda\right)^{1 / 3}-\left(\sqrt{\lambda^{2}+64 / 27}-\lambda\right)^{1 / 3}, \\
& a_{2}=-\frac{1}{4} a_{1}^{2}, \quad E_{g} / N_{p}=\lambda-a_{1}^{2}-a_{2}^{2} ; \operatorname{SUBl}(2) .
\end{aligned}
$$

Solutions for arbitrary $\mathrm{n}$ and $\lambda$ to the general SUBI(n) approximation to Eq. (40) are easily obtained numerically on a micro-computer. Convergence of the iterated solution is rapid. An accuracy of 6 significant figures, for example, is usually obtained in less than about 5 iterations. The g.s. energy per plaquette, $E_{g} / N_{p}$, is given in Fig. 2 and Table 1 as a function of $\lambda$ for several SUBI(n) schemes. The full SUB1 values, which are the exact solutions of the Mathieu equation (19), are actually well represented, to the level of accuracy shown, by the $\operatorname{SUBl}(20)$ results for the whole range of $\lambda$ displayed. The convergence with index $n$ of the SUB1(n) results to the SUB1 limit is clearly quite rapid, even in the large- $\lambda$ (weak-coupling) limit.

It is also very interesting to compare our SUBI(n) scheme results with the corresponding results for the Mathieu problem from perturbation theory in the strong-coupling $(\lambda \rightarrow 0)$ limit. $U p$ to eighth-order in this limit, the g.s. eigenvalue of Eq. (19) is given by, 45

$$
\varepsilon_{0} \underset{\lambda \rightarrow 0}{\longrightarrow} \lambda-\frac{1}{4} \lambda^{2}+\frac{7}{256} \lambda^{4}-\frac{29}{4608} \lambda^{6}+\frac{68687}{37748736} \lambda^{8}+O\left(\lambda^{10}\right) .
$$

We have explicitly verified that in this strong-coupling limit the SUB1(4) approximation for $E_{g} / N_{p}$ exactly reproduces this series to the order shown. In general, the $\lambda \rightarrow 0$ limiting form of the g.s. energy in SUBI(n) approximation 


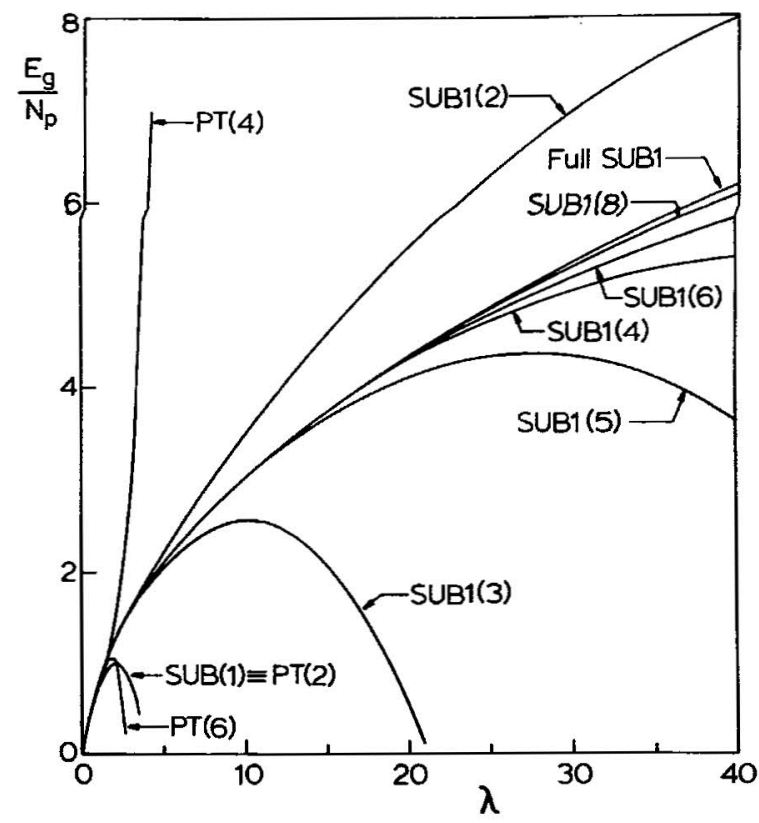

Fig. 2. Ground-state energy per plaquette, $E_{g} / N_{p}$, of the $U(1)$ gauge field model in (2+1)-dimensions, as a function of coupling constant, $\lambda$. Our full SUB1 approximation and various SUB1(n) sub-approximations are shown as well as some results of $n^{\text {th }}$-order strong-coupling $(\lambda \rightarrow 0)$ perturbation theory, PT(n).

exactly reproduces the result from (2n)th-order perturbation theory, PT(2n). Indeed, the SUBI(n) scheme actually provides a very efficient way to generate the coefficients of the terms in the perturbative treatment of the Mathieu problem! A more detailed comparison of the SUB1(n) and PT(2n) results for the g.s. energy is provided by Fig. 2, where we see clearly that the perturbative results are very poor for $\lambda \geq 1$.

Clearly, the range of validity of the SUB1(n) results extends to values well above $\lambda=1$, which is an approximate natural boundary for the PT(2n) results, even for relatively low values of $n$. The accuracy of different orders of perturbation theory as a function of $\lambda$ is shown more explicitly in Fig. 3. The accuracy, $\mathbb{A}$, of a quantity $E$ is, roughly speaking, the number of significant figures of the approximate result, $E_{\text {approx., compared with its }}$ exact counterpart, $E_{\text {exact }}$. It is defined more precisely as,

$$
A \equiv \log _{10}\left|\frac{E_{\text {exact }}}{E_{\text {exact }}-E_{\text {approx. }}}\right| .
$$

Figure 3 exhibits rather well the strongly non perturbative nature of the Mathieu problem, and the fact that the series of Eq. (46) has a finite radius of convergence.

By contrast, the accuracy of the comparable SUB1(n) results is displayed in the same way in Fig. 4. It is clear that these results represent a very natural extension of the $\mathrm{PT}(2 \mathrm{n})$ approximations. They comprise, in effect, a well-defined analytic continuation or resummation of the PT(2n) results, within the context of a systematic hierarchy of approximations. In this sense, the 


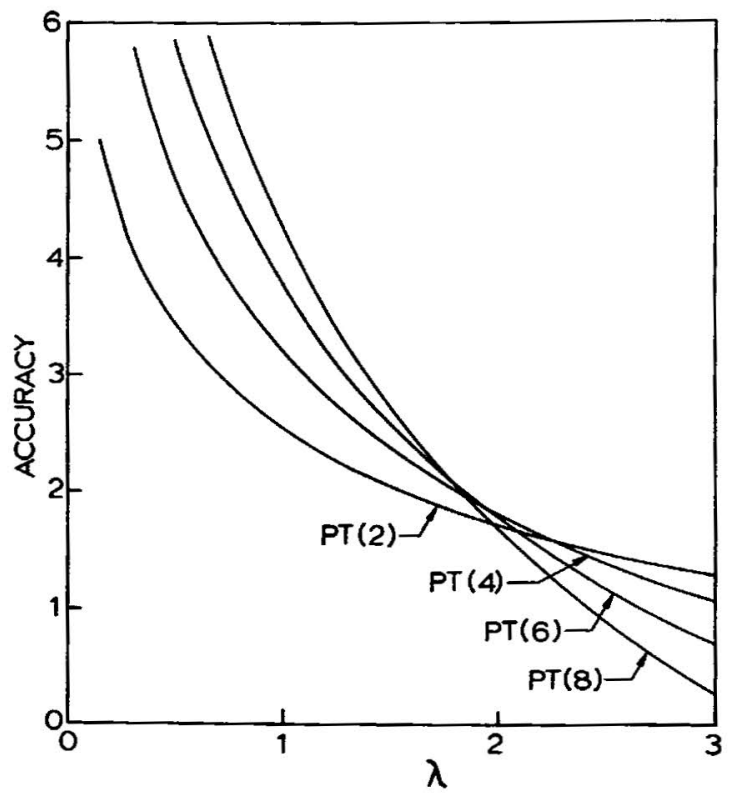

Fig. 3. The accuracy, defined in Eq. (47), as a function of coupling constant, $\lambda$, for the ground-state energy, $\varepsilon_{0}$, of the Mathieu equation (19) calculated in $n^{\text {th }}$-order strong-coupling $(\lambda \rightarrow 0)$ perturbation theory, $P T(n)$, for $n=2,4,6$, and 8 , compared to the exact result.

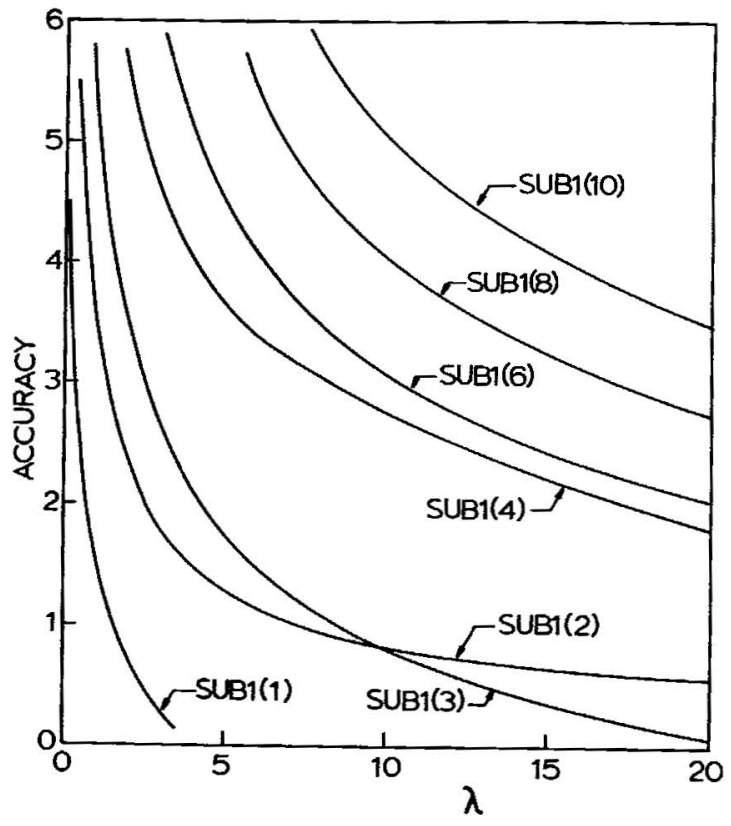

Fig. 4. The accuracy, defined in Eq. (47), as a function of coupling constant, $\lambda$, for the ground-state energy, $\varepsilon_{0}$, of the Mathleu equation (19) calculated in our SUB1(n) sub-approximation, for various values of $n$, compared to the exact result. 
SUBI(n) scheme scheme may be contrasted with alternative rather ad hoc approaches for extending the range of validity or the accuracy of similar PT(n) sequences. These include Padé approximant and related techniques.

In fact, the nonperturbative nature of the Mathieu equation has long made it a testing-ground for different techniques to attack lattice gauge theories. These have included variational methods, ${ }^{46}$ and use of the Lanczos algorithm ${ }^{37}$ to improve upon them. More generally, the U(1) lattice gauge model has also been investigated by such other methods as various Monte Carlo techniques, ${ }^{14,16,33}$ and the method of correlated basis functions. ${ }^{21}$ Most of these alternative techniques have demonstrated the necessity to include higherorder mode couplings. Our own results for the SUB1(n) scheme have clearly proven the efficacy of the CCM for handling the nonperturbative sector of the U(1) lattice gauge model.

\section{THE LSUBn SCHEME FOR THE $Z(2)$ MODEL}

We have given in Sec. 3 [and see Eqs. (31)-(33)] the (in principle) exact CCM forms for the ket and bra states of the $\mathrm{Z}(2)$ model. Although we could now in principle perform a SUBn sequence of approximations for the $Z(2)$ model as outlined above for the U(1) model, we prefer here to employ a different hierarchy of approximations, which we call the LSUBn sequence. This approximation scheme, which has no simple counterpart for continuous extended systems, has been recently developed within the context of quantum spin lattice models, ${ }^{28}$ where it has met with considerable success. It is particularly suited for treating lattice systems where the interaction forces are highly localized (i.e., short-ranged).

For our current 2D Z(2) model a given LSUBn approximation includes in the correlation operators $S$ and $\tilde{\mathbf{S}}$ all possible products of $\sigma_{3}(\ell)$ operators around closed (but not necessarily connected) contours which lie inside the closed, connected contours formed by all distinct groups of $n$ contiguous elementary plaquettes on the lattice. We consider here only the two simplest such approximations, namely LSUB1 and LSUB2. The former includes only the single contour corresponding to a single elementary plaquette of four links as in Fig. 1, whereas the latter includes the additional contour formed by a pair of neighbouring elementary plaquettes. More specifically we have,

$$
\begin{gathered}
\mathbf{S} \rightarrow \mathbf{S}_{\text {LSUB1 }}=\sum_{p} s_{1} U(p), \\
S \rightarrow S_{\text {LSUB2 }}=\sum_{p} s_{1} U(p)+\sum_{p} \sum_{\rho} s_{2} U(p) U(p+\rho),
\end{gathered}
$$

where $U(p)$, as defined by Eq. (30) is a product of $\sigma_{3}(\ell)$ operators around the links bordering the elementary plaquette $p$, and $p$ is one of the 4 lattice vectors connecting neighbouring elementary plaquettes on the $2 \mathrm{D}$ square lattice.

The correlation operator $\tilde{\mathbf{S}}$ is defined analogously in the two approximations [and see Eqs. (31)-(33)] by the replacements $s_{1} \rightarrow \tilde{s}_{1}, s_{2} \rightarrow \tilde{s}_{2}$. We note that since $\sigma_{3}^{2}=1$, the product of $U(p)$ operators for any set of contiguous elementary plaquettes is equivalent to a product of $\sigma_{3}(\ell)$ operators around the links forming their boundary.

The derivation of the CCM equations to determine the unknown cluster configuration coefficients now proceeds in the usual fashion. Thus, the g.s, 
energy expectation value, $\overline{\mathbf{H}}$, of Eq. (26) is first evaluated. The ket g.s. cluster coefficients, for example, are then obtained as in Eq. (27), by requiring $\overline{\mathrm{H}}$ to be stationary with respect to the corresponding bra g.s. coefficients. In order to evaluate $\overline{\mathbf{H}}$ we need to calculate the similarity transform, $\mathrm{e}^{-\mathrm{S}_{\mathrm{He}} \mathrm{S}}$. As usual, we utilize the well-known nested-commutator expansion,

$$
\mathrm{e}^{-\mathrm{S}_{\mathrm{He}} \mathrm{S}}=\mathbf{H}+[\mathbf{H}, \mathbf{S}]+\frac{1}{2 !}[[\mathrm{H}, \mathbf{S}], \mathbf{S}]+\cdots .
$$

However, whereas the infinite expansion of Eq. (49) usually terminates in practice for most standard many-body problems (and, more particularly, when $\mathbf{S}$ is composed only of creation operators with respect to the cyclic vector $|\Phi\rangle$, and $\mathrm{H}$ is a finite-order multinomial in the corresponding creation and destruction operators ${ }^{24}$ ), this is not the case here.

Nevertheless, for the $\mathrm{Z}(2)$ model under consideration, the non-terminating expansion of Eq. (49) is readily resummed. Thus, consider a general correlation operator $S$ of the form,

$$
\mathrm{S}=\sum_{\mathrm{p}} \sum_{\mathrm{c}} \mathrm{s}_{\mathrm{c}} \mathrm{U}_{\mathrm{c}}(\mathrm{p})
$$

where $U_{c}(p)$ is a product of $\sigma_{3}$ operators around a particular lattice contour of shape and orientation specified by the index $C$, and where the index $p$ labels some one particular plaquette in a specified ordering of the plaquettes inside C. We note that the c-number cluster amplitudes $\left\{s_{c}\right\}$ do not depend on the index $\mathrm{p}$ due to the lattice translational invariance. It is now straightforward to show that,

$$
\mathrm{e}^{-\mathrm{S}_{\mathrm{He}} \mathrm{S}}=-\sum_{\ell}\left[\sigma_{1}(\ell) \cosh \mathrm{G}-\mathrm{i}_{2}(\ell) \sinh \mathrm{G}\right]-\lambda \sum_{\mathrm{p}} \mathrm{U}(\mathrm{p}),
$$

where the operator $G$ is defined by,

$$
\mathrm{G} \equiv 2 \sum_{\mathrm{p} \ni \ell} \sum_{\mathrm{c}} \mathrm{s}_{\mathrm{c}} \mathrm{U}_{\mathrm{c}}^{\prime}(\mathrm{p} ; \ell)
$$

wherein the sum over plaquettes is restricted to those which include the link $\ell$, and where $U_{c}^{\prime}(p ; \ell)$ is identical to the product of $\sigma_{3}$ operators comprising $U_{c}(p)$ except that the single operator $\sigma_{3}(\ell)$ on the particular link $\ell$ is omitted and the ordering of the plaquettes inside $C$ is relaxed. Both the terms cosh $G$ and sinh $G$ in Eq. (51) can then be expanded using the usual expansion rules for $\cosh (A+B)$ and $\sinh (A+B)$. Finally, using that $\left[U_{C}^{\prime}(p ; l)\right]^{2}=1$ for all $C, p$ and $\ell$, since $\sigma_{3}^{2}=1$, we have the relations,

$$
\left.\cosh \left[\mathrm{s}_{\mathrm{c}} \mathrm{U}_{\mathrm{c}}^{\prime}(\mathrm{p} ; \ell)\right]=\cosh \mathrm{s}_{\mathrm{c}} ; \sinh \left[\mathrm{s}_{\mathrm{c}} \mathrm{U}_{\mathrm{c}}^{\prime}(\mathrm{p} ; \ell)\right]=\mathrm{U}_{\mathrm{c}}^{\prime}(\mathrm{p} ; \ell)\right] \sinh \mathrm{s}_{\mathrm{c}},
$$

which enable us ultimately to rewrite Eq. (51) in a form in which the hyperbolic functions act only on the c-number amptitudes $\left\{s_{c}\right\}$. 
We quote only the final result for $\overline{\mathrm{H}}$ for the $2 \mathrm{D} \mathrm{Z}(2)$-model in the LSUB1 approximation,

$$
\overline{\mathrm{H}}_{\mathrm{LSUB1}}=-\sum_{\ell}\left[\cosh ^{2}\left(2 \mathrm{~s}_{1}\right)+\frac{1}{2} \widetilde{\mathrm{s}}_{1}\left\{\lambda-2 \sinh \left(4 \mathrm{~s}_{1}\right)\right\}\right] \text {, }
$$

where, in writing the result as a sum over link variables only, we have used that $\mathrm{N}_{\ell}=2 \mathrm{~N}_{\mathrm{p}}$ for the infinite $2 \mathrm{D}$ square lattice. By making $\overrightarrow{\mathrm{H}}$ stationary with respect to $\widetilde{s}_{1}$ and $s_{1}$, we readily find,

$$
s_{1}=\frac{1}{4} \sinh ^{-1}\left(\frac{1}{2} \lambda\right), \quad \widetilde{s}_{1}=\frac{1}{2} \lambda\left(1+\frac{1}{4} \lambda^{2}\right)^{-1 / 2} ; \text { LSUB } 1,
$$

and the stationary value of the g.s. energy per link is given as,

$$
E_{g} / N_{\ell}=-\frac{1}{2}\left(1+\sqrt{1+\frac{1}{4} \lambda^{2}}\right) ; \text { LSUBI. }
$$

The comparable equations which determine the ket-state coefficients $\left(s_{1}, s_{2}\right)$ and the g.s. energy per link in the LSUB2 approximation may also be derived after some algebra as,

$$
\begin{gathered}
\lambda+\sinh \left(2 s_{1}\right) \cosh \left(2 s_{1}\right)\left[12 \sinh \left(2 s_{2}\right)-4 \cosh \left(2 s_{2}\right)\right] \cosh ^{5}\left(2 s_{2}\right)=0, \\
\sinh ^{2}\left(2 s_{1}\right)\left[2 \sinh ^{2}\left(2 s_{2}\right)+\cosh ^{2}\left(2 s_{2}\right)\right]-6 \cosh ^{2}\left(2 s_{1}\right) \sinh \left(2 s_{2}\right) \cosh \left(2 s_{2}\right)=0, \\
E_{g} / N_{\ell}=-\cosh ^{2}\left(2 s_{1}\right) \cosh ^{6}\left(2 s_{2}\right) ; \text { LSUB2. }
\end{gathered}
$$

Equations (57) are readily solved numerically and the solution for $E_{g} / N_{\ell}$ is shown in Fig. 5 together with the LSUB1 result from Eq. (56).

$A$ priori, we expect the above LSUBn approximations to be most accurate in the strong-coupling $(\lambda \rightarrow 0)$ regime, where we may compare with the known perturbation-theory results for the $2 \mathrm{D} Z(2)$-model, ${ }^{39}$

$$
\frac{E_{g}}{N_{\ell}} \underset{\lambda \rightarrow 0}{\longrightarrow}-1-\frac{\lambda^{2}}{16}-\frac{\lambda^{4}}{3072}-\frac{\lambda^{6}}{196608}+O\left(\lambda^{8}\right)
$$

Our LSUB1 result of Eq. (56) is clearly seen to agree with Eq. (59) to $2^{\text {nd }}$ order. An analytic small- $\lambda$ expansion of Eqs. (57)-(58) also shows that our LSUB2 result reproduces the result of $4^{\text {th }}$-order perturbation theory. Although we have not attempted a strict proof, we expect that (suitably constructed) LSUBn approximations with $n>2$ will also reproduce the results of $(2 n)^{\text {th-order }}$ perturbation theory, PT(2n).

It is also interesting to compare the weak-coupling $(\lambda \rightarrow \infty)$ limits of our LSUB1 and LSUB2 results with the corresponding results from perturbation theory in this regime, ${ }^{39}$

$$
\frac{E_{g}}{N_{\ell}} \underset{\lambda \rightarrow \infty}{\longrightarrow}-\frac{1}{2} \lambda\left[1+\frac{1}{2} \lambda^{-2}+\frac{5}{32} \lambda^{-4}+O\left(\lambda^{-6}\right)\right] .
$$

As expected, we do not get agreement in this limit. Nevertheless, both LSUB1 and LSUB2 approximations give the correct leading asymptotic behaviour, namely 
linear in $\lambda$, albeit with an incorrect coefficient. More precisely, Eq. (56) trivally gives $E_{g} / \mathrm{N}_{\ell} \rightarrow-0.25 \lambda$ as $\lambda \rightarrow \infty$ in the LSUB1 approximation, whereas an analytic large- $\lambda$ expansion of Eqs. (57)-(58) shows that the leading-order LSUB2 result is $E_{g} / N_{\ell} \rightarrow-k \lambda$ as $\lambda \rightarrow \infty$, where $k=(7+3 \sqrt{7}) / 28 \approx 0.533$. This latter value is rather close to the exact value, $\mathrm{k}=0.5$.

Finally, we note that for the 2D $\mathrm{Z}(2)$-model Cardy and Hamber 47 and also Suranyi ${ }^{48}$ have proposed a trial variational g.s. wave function of precisely our LSUB1 form,

$$
|\Psi\rangle=\exp \left[\mathrm{s}_{1} \underset{\mathrm{p}}{{ }_{\mathrm{p}} \mathrm{U}(\mathrm{p})}\right]|\Phi\rangle
$$

It is not difficult to show that if the expectation value of the Hamiltonian of Eq. (15) is evaluated in this trial state in the usual fashion as $E_{8}=$ $\langle\Psi|H| \Psi\rangle /\langle\Psi \mid \Psi\rangle$, where the bra state is now the explicit Hermitian adjoint of the ket state, we find

$$
E_{g} / N_{\ell}=-\operatorname{sech}^{2}\left(2 s_{1}\right)-\frac{1}{2} \lambda \tanh \left(2 s_{1}\right)
$$

This expression may be minimized with respect to $s_{1}$ to find the simple variational result,

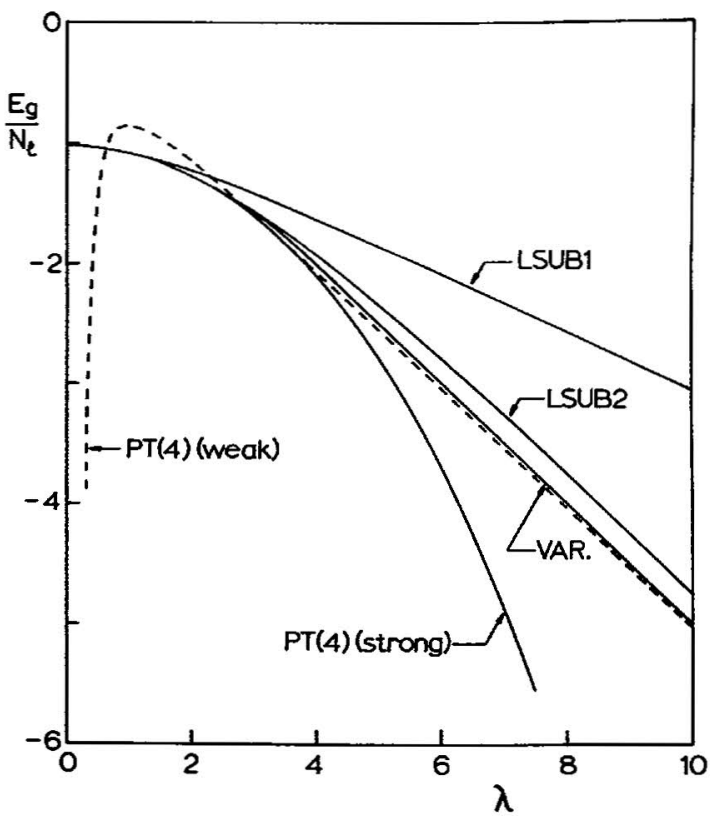

Fig. 5. Ground-state energy per link, $E_{g} / N_{\ell}$, of the $Z(2)$ gauge model on a $2 D$ square lattice, as a function of the coupling constant, $\lambda$. Shown are our LSUB1 and LSUB2 results; the variational (VAR) result of Eq. (63); and the fourth-order perturbation theory, PT(4), results in both the strong-coupling $(\lambda \rightarrow 0)$ and weak-coupling $(\lambda \rightarrow \infty)$ limits, from Eqs. (59) and $(60)$ respectively. 


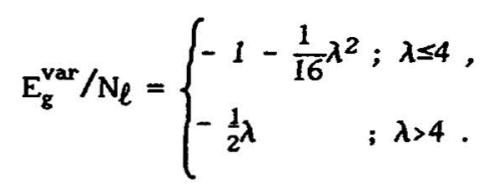

Like our CCM LSUB1 approximation this also reproduces the 2nd-order strongcoupling $(\lambda \rightarrow 0)$ perturbation theory result of Eq. (59). However, unlike our LSUB1 result, this variational treatment both gives the exact leading asymptotic behaviour in the weak-coupling $(\lambda \rightarrow \infty)$ limit, and gives an indication of a (second-order) phase transition at a critical value $\lambda_{c}=4$, which may be compared with the more precise value $\lambda_{c} \approx 3.1$.

The above simple variational approximation is clearly superior (at least so far as the g.s. energy is concerned) to our LSUB1 result. Interestingly, unlike the more general situation (including the U(1) case) where this variational approach is not easily extended to larger classes of trial wave functions without making further approximations in the evaluation of the energy expectation value (which then destroy the variational upper bound), this is not so for the $\mathrm{Z}(2)$ model on the $2 \mathrm{D}$ square lattice. The underlying simplicity of this model has, for example, enabled Dagotto and $\mathrm{Moreo}^{49}$ to use larger classes of trial wave functions in exactly the spirit of our LSUBn scheme. For example, for our LSUB2 wave function of Eq. (48b) considered as a trial variational state, they now find a phase transition in the g.s. energy at a critical value, $\lambda_{c}=3.85$. The interested reader is referred to Ref. [49] for further details.

We stress, however, that for more general lattice gauge field theories, one cannot evaluate the g.s. energy expectation value, $E_{g}=\langle\Psi|H| \Psi\rangle /\langle\Psi \mid \Psi\rangle$, without making further (largely uncontrolled) approximations, for the classes of wave functions $\{|\Psi\rangle\}$ which one can handle exactly by our CCM techniques. As usual, ${ }^{24}$ the biorthogonal $\mathrm{CCM}$ formulation can continue to take advantage of the underlying similarity transformation to evaluate $\overline{\mathbf{H}}$ as in Eq. (26), when this is not practicable in the ordinary variational method.

\section{SUMMARY AND DISCUSSION}

In the present article we have concentrated, largely for pedagogical reasons, on the ground-state energy eigenvalue and ket eigenfunction. We have, for similar reasons, also focused attention on relatively low-level CCM approximations in both the SUBn sequence which at all orders retains some correlations of infinite range, and the LSUBn sequence which is more geared to the inclusion of short-range correlations. It should be immediately apparent to the reader how to derive the analogous bra ground-state equations, and hence how to calculate g.s. expectation values of arbitrary operators. In this context we shall be especially interested to calculate the physically interesting 2-plaquette correlation functions that become meaningful when we proceed beyond the SUB1 level of approximation used here for the U(1) model, for example. Furthermore, just as in the particular case of the spin-lattice problems studied by CCM techniques, ${ }^{28}$ it is also quite straightforward to implement the standard CCM technology to study the excited states ${ }^{24}$ of our lattice gauge models.

For the 2D $\mathrm{Z}(2)$ model studied here we are particularly interested to study the phase transition which occurs at a critical coupling. The localized LSUBn sequence within our standard CCM approach shows no obvious sign of a transition in the g.s. energy although, interestingly, the variational approach based on 
the same class of wave functions does. ${ }^{49}$ It will be of interest to study this matter further within the CCM framework, perhaps by requiring that the model state $|\Phi\rangle$ of Eq. (29) be redefined in terms of a product of more general maximum-overlap link orbitals involving an additional (rotation) parameter that can be chosen to maximize the overlap of the new model state $\left|\Phi^{\prime}\right\rangle$ with the true state $\left|\Psi_{0}\right\rangle$. We note in this regard that Kümmel ${ }^{50}$ has investigated the sufficiency condition for such maximum-overlap orbitals actually to maximize the overlap rather than simple to extremize it, as given by the usual necessary condition. He shows in particular how the extra sufficency condition can be utilized to identify within the CCM the onset of a critical "shape instability" which marks the transition to a different optimal basis, or configuration, as some appropriate internal parameter (here, $\lambda$ ) is varied.

The great attraction of the CCM is, as we have already remarked, that it is truly a universal method. Hence, motivated by the preliminary studies reported here, we intend to extend the application of CCM techniques to, for example: (i) the more general $\mathrm{Z}(\mathrm{N})$ model discussed in Sec. 2.2, particularly to investigate in detail the $\mathrm{N} \rightarrow \infty$ limit to $\mathrm{U}(1)$; (ii) both the $\mathrm{Z}(2)$ and $\mathrm{U}(1)$ models considered here, in (3+1)-dimensions; and (iii) such non-Abelian gauge theories as SU(2). We hope to report on these calculations elsewhere.

\section{ACKNOWLEDGEMENT}

One of us (RFB) acknowledges support for this work in the form of a research grant from the Science and Engineering Research Council (SERC) of Great Britain.

\section{REFERENCES}

1. K.G. Wilson, Phys. Rev. D 10:2445 (1974).

2. J. Kogut and L. Susskind, Phys. Rev. D 11:395 (1975).

3. S. Elitzur, Phys. Rev. D 12:3978 (1975).

4. A.M. Polyakov, Phys. Lett. B72:477 (1978).

5. E. Fradkin and L. Susskind, Phys. Rev. D 17:2637 (1978).

6. S.D. Drell, H.R. Quinn, B. Svetitsky and M. Weinstein, Phys. Rev. D 19:619 (1979).

7. R. Balian, J.M. Drouffe and C. Itzykson, Phys. Rev. D $\underline{10}: 3376$ (1974); 11:2098 (1975); 11:2104 (1975).

8. D. Horn, M. Weinstein and S. Yankielowicz, Phys. Rev. D 19:3715 (1979).

9. F.J. Wegner, J. Math. Phys. 12:2259 (1971).

10. S.F. Edwards and P.W. Anderson, J. Phys. F $\underline{5}: 965$ (1975).

11. A.A. Migdal, Sov. Phys.-JETP 42:413, 743 (1976).

12. M. Creutz, "Quarks, Gluons and Lattices," Cambridge University Press (1983).

13. M. Creutz, L. Jacobs and C. Rebbi, Phys. Rep. 95:201 (1983).

14. D.W. Heys and D.R. Stump, Phys. Rev. D 28:2067 (1983).

15. S.A. Chin, J.W. Negele and S.E. Koonin, Ann. Phys. (NY) 157:140 (1984).

16. T. Barnes and D. Kotchan, Phys. Rev. D 35:1947 (1987).

17. H.H. Roomany and H.W. Wyld, Phys. Rev. D 21:3341 (1980).

18. A.C. Irving and A. Thomas, Nucl. Phys. B200 [FS4]:424 (1982).

19. A.C. Irving, J.F. Owens and C.J. Hamer, Phys. Rev. D 28:2059 (1983).

20. M.D. Kovarik, J.W. Darewych and R. Koniuk, Phys. Rev. D 33:3654 (1986).

21. A. Dabringhaus, M.L. Ristig and J.W. Clark, Phys. Rev. D 43:1978 (1991).

22. J.W. Clark and E. Feenberg. Phys. Rev. 113:388 (1959); H.W. Jackson and E. Feenberg, Ann. Phys. (NY) 15:266 (1961).

23. F. Coester, Nucl. Phys. $7: 421$ (1958); F. Coester and H. Kümmel, Nucl. Phys. 17:477 (1960). 
24. R.F. Bishop, Theor. Chim. Acta 80:95 (1991).

25. K.G. Wilson, Nucl. Phys. B (Proc. Suppl.) 17:82 (1990).

26. R.J. Bartlett, Theor. Chim. Acta 80:71 (1991) -- and note also that issues 2-6 of this volume are entirely devoted to articles on the coupled cluster theory of electron correlations in many-electron systems.

27. M. Roger and J.H. Hetherington, Phys. Rev. B 41:200 (1990).

28. R.F. Bishop, J.B. Parkinson and Yang Xian, Phys. Rev. B 43:13782 (1991); 44:9425 (1991); Theor. Chim Acta 80:181 (1991).

29. V. Alessandrini, V. Hakim and A. Krzywicki, Nucl. Phys. B200[FS4]:355 (1982).

30. J. Villain, J. Phys. (Paris) 36:581 (1975).

31. M. Göpfert and G. Mack, Commun. Math. Phys. 82:545 (1981).

32. J. Ambjørn, A.J.G. Hey and S. Otto, Nucl. Phys. B210[FS6]:347 (1982).

33. G. Bhanot and M. Creutz, Phys. Rev. D 21:2892 (1980).

34. U.M. Heller, Phys. Rev. D 23:2357 (1981).

35. D.W. Heys and D.R. Stump, Nucl. Phys. B257:19 (1985); B285:13 (1987).

36. J.W. Choe, A. Duncan and R. Roskies, Phys. Rev. D 37:472 (1988).

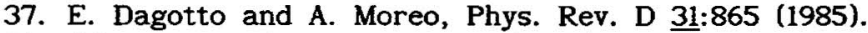

38. J.B. Kogut, Phys. Rep. 67:67 (1980).

39. P. Pfeuty and R.J. Elliot, J. Phys. C $4: 2370$ (1971).

40. J. Kogut and D. Sinclair, Phys. Lett. 81A:149 (1981).

41. E. Fradkin and S. Raby, Phys. Rev. D 20:2566 (1979).

42. T. Banks and A. Zaks, Nucl. Phys. B200[FS4]:391 (1982).

43. D. Bessis and M. Villani, J. Math. Phys. 16:462 (1975).

44. D. Robson and D.M. Webber, Z. Phys. C 7:53 (1980).

45. G. Blanch, in: "Handbook of Mathematical Functions," M. Abramowitz and I.A. Stegun (eds.), N.B.S. Appl. Math. Ser. 55, U.S. Govt. Printing Office, Washington, D.C. (1964), p.721.

46. W. Langguth, Z. Phys. C 23:289 (1984).

47. J. Cardy and H. Hamber, Nucl. Phys. B170[FS1]:79 (1980).

48. P. Suranyi, Nucl. Phys. B210:519 (1982).

49. E. Dagotto and A. Moreo, Phys. Rev. D 29:300 (1984).

50. H.G. Kümmel, Nucl. Phys. A317:199 (1979). 